\title{
Aprendizaje e innovación en las organizaciones de la sociedad civil y en la universidad asociadas en programas de extension universitaria
}

\author{
Adriana Norma Fassio, María Gabriela Rutty, Carla Maroscia
}

Resumen: Se exponen los resultados de la investigación realizada en la Facultad de Ciencias Económicas de la Universidad Nacional de La Plata (FCE UNLP) denominada "Aprendizaje organizacional en organizaciones de la sociedad civil de la ciudad de La Plata y zona de influencia que llevan adelante programas vinculados a la innovación social responsable". Se presentan los aprendizajes organizacionales reconocidos por las organizaciones de la sociedad civil (OSC) de la ciudad de La Plata y por las cátedras de la Facultad que brindaron asistencia técnica en esta instancia asociativa.

Palabras clave: aprendizaje 100204; organizaciones no gubernamentales 050303

Abstract: This study reports the results of the research project "Organizational learning in civil society organizations that implement programs based on responsible social innovation in the city of La Plata and its area of influence", which was carried out at the Faculty of Economic Sciences of the National University of La Plata (FCE $U N L P$ ). The study presents the organizational learning described by the civil society organizations of the city of La Plata and by the faculty chairs that provided technical assistance during this collaboration.

Keywords: Learning 100204; Non-governmental organizations 050303 


\section{Planteamiento del problema}

En un contexto de crisis social y económica como el actual en la Argentina, las organizaciones de la sociedad civil (OSC) brindan servicios a la comunidad complementando u ocupando los espacios vacantes de la política social estatal. En el proceso de implementación de innovaciones es frecuente que las organizaciones adquieran nuevos conocimientos como resultado de la necesidad de resolver los problemas que surgen de la práctica. Estos aprendizajes no siempre son registrados y se integran a la cultura de las organizaciones de manera inadvertida. En este artículo se exploran los aprendizajes organizacionales realizados a partir de una experiencia innovadora en la cual la universidad brinda una capacitación en gerencia pública a OSC a través del área de extensión universitaria.

En la Argentina es escasa la literatura sobre procesos de innovación y aprendizaje (AO) en las OSC que desarrollan su actividad en sectores vulnerables. Asimismo, el análisis bibliográfico muestra que el trabajo de sistematización y evaluación de las experiencias innovadoras suele omitirse en el ámbito universitario (Alvarez, 2015). Por lo tanto, la detección de cambios y aprendizajes ocurridos en las organizaciones que han recibido capacitación vía la extensión universitaria de la facultad es una estrategia diagnóstico-evaluativa de suma importancia para plantear propuestas de mejora no solo en las OSC estudiadas, sino también en las futuras acciones de fortalecimiento por parte de la universidad pública, cuyo resultado se objetiva en la mejora de la calidad de vida de la población a la cual prestan sus servicios.

\section{Objeto de estudio}

En este artículo se presentan los resultados de la investigación realizada en la Facultad de Ciencias Económicas de la Universidad Nacional de La Plata (FCEUNLP) denominada "Aprendizaje organizacional en organizaciones de la sociedad civil de la ciudad de La Plata y zona de influencia que llevan adelante programas vinculados a la innovación social responsable". Se indaga sobre los aprendizajes, cambios e innovaciones organizacionales reconocidos tanto por OSC de la ciudad de La Plata como por las cátedras que participan en las capacitaciones en el marco del programa de extensión universitaria "Aprendizaje en acción. Fortaleciendo la gestión de las OSC de la ciudad de La Plata", llevado a cabo desde el año 2015 por docentes de dicha casa de estudios.

Estos aprendizajes son producto de las alianzas entre la extensión universitaria de la FCE- UNLP y las OSC del territorio y constituyen espacios de cogestión entre la universidad y la comunidad a partir de las demandas explicitadas por las organizaciones y/o actores y la resignificación que se hace desde la universidad. 


\section{Fundamentación teórica}

Las OSC se orientan a la consecución de diversos fines: defensa de intereses mutuos de base territorial, asistencia, investigación, promoción y desarrollo, y defensa de derechos. Si bien ha habido cambios recientes en las formas de inserción de las OSC como consecuencia de la crisis de los partidos políticos, de los sindicatos y del modelo de integración social en la Argentina del nuevo milenio, sus formas de gestión, vulnerabilidades y fortalezas no se han modificado (De Piero, 2020). Es decir, a pesar de la diversidad de cometidos, en líneas generales las OSC siguen compartiendo ciertas problemáticas comunes respecto de la carencia o discontinuidad de recursos, de profesionales y de armado institucional, entre otras identificadas hace ya más de dos décadas (Suárez, 1995).

Respecto de las debilidades presentes en estas organizaciones, retomamos el concepto de vulnerabilidad organizacional de Suárez (1995), considerado como una debilidad constitucional en puntos críticos que implican dificultades para el cumplimiento de los objetivos básicos de la organización, anclados en su misión y visión. A partir de ella se explicarían los períodos de "latencia" organizacional a la espera de acceder nuevamente a recursos que le permitan llevar adelante acciones en pro de la consecución de los objetivos planificados.

El grado de vulnerabilidad es analizado a través de tres dimensiones básicas: a) profesionalización incompleta; $b$ ) institucionalización precaria, y c) financiamiento discontinuo.

Las OSC ponen en marcha innovaciones que se enfrentan la mayoría de las veces con los límites de recursos escasos y con todos los problemas conexos que esto supone (Fassio, 2007). Como se señaló más arriba, esas innovaciones pueden generar aprendizajes en las organizaciones que suelen ser invisibles (Asociación Española de Contabilidad y Administración de Empresas. AECA, 2011) y que inadvertidamente pasan a formar parte de la cultura de la organización. Identificar estos saberes y los procesos que inauguran permite a las organizaciones evaluarlos para impulsar el desarrollo organizacional. Su identificación también permite, al compartirlos, dado que surgen de la resolución de problemas, la generación de un circuito de aprendizaje y/o la formación de un repertorio de buenas prácticas.

Adicionalmente, es necesario considerar que la innovación debe orientarse por ciertos principios que hagan posible que su puesta en marcha impacte positivamente en la calidad de vida de las personas y en el ejercicio de sus derechos. Así, las iniciativas de "innovación social responsable" comprenden tres dimensiones fundamentales: la satisfacción de las necesidades humanas (dimensión de contenido); cambios en las relaciones sociales, especialmente con respecto a la 
gobernanza (dimensión de proceso); y aumento de la capacidad sociopolítica y del acceso a los recursos (dimensión de empoderamiento) (Comisión Europea, 2013; Gerometta, Haussermann y Longo, 2005; Grunwald, 2011; Moulaert y Sekia, 2003; Rodríguez y Cedeño, 2012; Schmidpeter, 2013).

En este estudio hemos abordado el concepto de aprendizaje organizacional (en sentido amplio), con la intención de discriminar hechos, o resultados (los aprendizajes: capacidades, conocimientos, posicionamientos, estrategias, etc.), los procesos (el recorrido por el cual un conocimiento es generado, compartido y luego integrado a las prácticas organizacionales, es decir, la dimensión dinámica del fenómeno) y las condiciones (relativas a la cultura como facilitadora del cambio). Nuestra hipótesis es que la innovación constituye la mejor oportunidad para generar nuevo aprendizaje organizacional (Fassio y Rutty, 2017, 2020).

La caracterización de los aprendizajes producidos se realizó sobre la base de algunos de los criterios desarrollados por AECA (2011): el grado de radicalidad del aprendizaje (cuán importante es para la organización), su orientación (conceptual u operativa), los niveles organizativos implicados, el grado de conciencia que los actores tienen de haber aprendido, la intencionalidad (aprendizaje formal o informal), voluntariedad y sistematización, y la fuente (externa-interna).

Los debates y argumentos sobre los diversos aspectos que involucran el aprendizaje y la gestión del conocimiento en las organizaciones dan lugar a discusiones de distintos autores que intentan proponer una visión multiparadigmática (Antonello y Godoy, 2010). Los desarrollos recientes en el campo del aprendizaje organizacional avanzan sobre la naturaleza colectiva del fenómeno (Antonacopoulou, 2006a, 2006b; Gore, 2003; Wenger, 2000), en oposición a visiones más clásicas que lo describen como un proceso individual que luego se comparte (Argyris, 1999; Brandi y Bente, 2011; Senge, 2005). Otro de los asuntos analizados en relación con la innovación organizacional son las condiciones de aprendizaje (Castañeda, 2015), y se señala como prioritario que las organizaciones puedan identificar estos mecanismos y operar sobre ellos en un sentido positivo a fin de generar los cambios necesarios para mejorar el desempeño organizacional (Gore y Manzini, 2010; Perlo, 2011).

Con relación a los aprendizajes organizacionales que se producen en el marco de organizaciones en contextos vulnerables, solo se han estudiado a partir del fenómeno de la constitución de redes y alianzas como un factor clave para la construcción de capacidad institucional (Liebler y Ferri, 2004). La construcción de alianzas con otras organizaciones se manifiesta como el camino para alcanzar nuevos aprendizajes y mejorar la organización, por medio del rescate y la incorporación de lo adquirido en experiencias previas de otras organizaciones 
(Bustinza, Molina y Arias-Aranda, 2010). Cabe señalar que la mayor parte de la literatura sobre alianzas toma a la empresa como unidad de estudio y su análisis se relaciona con las mejoras en la productividad y potencial rentabilidad, y es muy poco abordado tanto para el sector público como para el universo de OSC. Otro concepto que se asocia al aprendizaje en relación con el contexto es el de "capacidad dinámica" (Dynamic capability) ${ }^{1}$, dado que el conocimiento colaborativo es un tipo específico de conocimiento adquirido por las organizaciones que influye tanto en su presente como en su futuro, y tiene un efecto directo positivo sobre el desarrollo de todos los procesos llevados a cabo en ellas.

El tema de redes y la gestión de alianzas constituye un interesante campo de estudio (Bustinza, Molina et al., 2010) especialmente vinculado a contextos vulnerables (Forni, 2002; Forni et al., 2012; Lieber y Ferri, 2004; Longo y Forni, 2004). Entre sus ventajas, Forni (2002:9) señala que "favorecen el aprendizaje, ya que posibilitan la gestación de síntesis novedosas de la información, cualitativamente distinta de la existente en cada uno de los nodos que la integran".

\section{Diseño y metodología}

Se tomó como población a estudiar las OSC vinculadas al programa "Aprendizaje en acción. Fortaleciendo la gestión de las OSC de la ciudad de La Plata" (implementado por la Secretaría de Extensión Universitaria [SEU] de la FCE UNLP), cuyo objetivo es brindar capacitación no formal en temas de gestión por parte de las unidades académicas de la FCE a partir de demandas realizadas por las OSC del Gran La Plata, a través de sus docentes, investigadores, alumnos y no docentes.

A partir de ello se conformó una muestra teórica de nueve organizaciones de la sociedad civil de la ciudad de La Plata que habían participado en más de un curso brindado por la SEU entre los años 2015 y 2017.

El diseño de la investigación es de un estudio de caso múltiple comparado con abordaje cualitativo (De Souza Bido, Bertoia da Silva, Teixeira Maggi da Silva y Reatto, 2015; De Souza Minayo, 2017; Forni 2010, 2012; Neiman y Quaranta, 2007; San Martín Cantero, 2014; Schmidt Godoy; Stake, 2013), y la muestra fue cerrada por saturación teórica.

Durante el año 2018 en cada una de las OSC se realizaron entrevistas semiestructuradas a los actores relevantes (informantes clave que habían participado

1 El concepto de Dinamic capability es entendido de acuerdo con el análisis y descripción que realizan Vera, Crossan y Appaydin (2011). El planteamiento es que la ventaja de la organización se deriva no del recurso específico, sino de la capacidad de la organización para crear continuamente, integrar y reconfigurar nuevos recursos. La capacidad dinámica es el proceso por el cual la organización usa recursos y alcanza nuevas configuraciones en los recursos. 
en las capacitaciones), así como observación participante de las actividades concernientes a la ejecución de los programas implementados por ellas. Se obtuvo un material de aproximadamente doce horas de duración que fue desgrabado, el cual, junto con las notas de campo (registro de observaciones), fue procesado con Atlas.ti.

Los ejes centrales de la indagación versaron sobre las problemáticas que la organización enfrentaba en el momento de realizar la capacitación en el marco de la asistencia técnica, los conocimientos que consideraron más valiosos, la capacidad de institucionalizarlos, la implementación de nuevas prácticas, y los problemas que afrontaron y las formas de solucionarlos, así como los obstáculos que se presentaron e impidieron llevar a cabo las innovaciones propuestas.

Asimismo, a finales de 2018 se realizaron seis entrevistas no estructuradas de aproximadamente una hora de duración a docentes y funcionarios de la Facultad de Ciencias Económicas de la UNLP que participaron en las capacitaciones del proyecto "Aprendizaje en acción" a través de sus respectivas cátedras, entrevistas que fueron transcritas y procesadas con Atlas.ti. En ellas se indagó sobre los aprendizajes organizacionales que realizaron para sí mismas la universidad o la cátedra como organización encargada de implementar la capacitación y sobre los procesos de aprendizaje en las OSC asistidas en el marco de la propuesta de aprendizaje en alianza.

\section{Principales resultados}

La extensión universitaria contactó con las organizaciones estudiadas para su inclusión en la capacitación del proyecto "Aprendizaje en acción" de diverso modo: por vínculos/invitación con algún miembro de la comunidad académica de la facultad, por búsqueda en la web y por difusión que realiza la facultad a partir de su base de datos de organizaciones de la sociedad civil.

\subsection{Problemas que afrontaba la OSC en el momento de realizar la capacitación}

La capacitación estuvo orientada a fortalecer las prácticas de gestión de las OSC. Las organizaciones, al ser consultadas sobre los principales problemas que detectan en su funcionamiento, hacen referencia a dificultades relacionadas con la Administración (cumplimiento de disposiciones administrativas para la obtención y rendición de subsidios públicos); la obtención de financiamiento; la comunicación tanto interna (trabajo en equipo; articulación de profesionales y voluntarios) como externa (relación con donantes); la incapacidad/desconocimiento de las 
técnicas para la elaboración de proyectos a partir de los cuales obtener apoyo estatal/donantes; el desconocimiento de técnicas de planificación; y la necesidad de conocer cómo resuelven los problemas otras organizaciones de éxito en el territorio. A partir de todo ello surge fuertemente la demanda del trabajo en red.

Para mí, algo puntual era el trabajo en red con otras instituciones. Entonces, a veces en nuestros espacios se nos generan muchas relaciones, más allá de que la organización es muy grande y tiene un área de comunicaciones. Uno siempre aprende de la escucha de otros espacios. El intercambio es fundamental. Además, porque nosotros somos una ONG que trabaja en red. Es imposible trabajar sin otros espacios. El conocimiento de ese tipo de instituciones es muy importante. (OSC 3) Compartir estos problemas y las soluciones con otras OSC a modo de red.

Identifiqué la necesidad de contar con herramientas para llevar adelante el proyecto ambiental, que es el área de interés mío y mi formación básica es la ecología, entonces yo tenía interés de llevar adelante un programa de medio ambiente y me daba cuenta de que faltaban herramientas como es la planificación de un proyecto. El curso este de aprendizaje en acción nos capacitó, nos dio herramientas, todo lo que hace a la cuestión de la gestión, de la planificación, de armado de un proyecto; y esas herramientas eran las que a mí me faltaban, entonces al detectar esa necesidad fue que me acerqué al curso. (OSC 6) Herramientas de planificación.

Los problemas generales mencionados están vinculados con las vulnerabilidades comunes a las OSC, como ya se dijo, debido al problema de escasez de recursos, "profesionalización incompleta e institucionalización precaria" (Suárez, 1995). Varios de los interlocutores señalan el trabajo en red como una estrategia que resulta oportuno promover. Las redes interorganizacionales son "conjunto seleccionado, persistente y estructurado de organizaciones autónomas que se vinculan para crear productos o servicios, sobre la base de contratos implícitos o explícitos" (Sulbrandt, Navarrete y Piergentilli, 2007: 153). De acuerdo con los expertos, los beneficios percibidos de las redes son abundantes, entre ellos el acceso a la información, experiencia y recursos financieros, incremento de la eficiencia y/o la posibilidad de reconstruir los lazos quebrados en momentos de crisis (Lieber y Ferri, 2004).

\subsection{Los conocimientos que se consideraron valiosos}

Las organizaciones han valorado positivamente los conocimientos obtenidos en los cursos. No obstante, señalan que existen déficits importantes en algunos ámbitos: la planificación estratégica a fin de cumplir los objetivos organizacionales; 
la división del trabajo; la elaboración de perfiles; el conocimiento de la normativa que las rige; las acciones a implementar; etc. También identifican la fortaleza de los valores que las guían. Por otro lado, y desde la perspectiva de la universidad, se alerta que el dictado de los cursos no es suficiente para que las OSC incorporen esos conocimientos, sino que solo constituye un muy buen punto de partida $y$, por lo tanto, se requiere establecer un nuevo dispositivo de apoyo. En este sentido, los actores involucrados destacan o manifiestan la necesidad de que el Estado los apoye y promueva.

A mí me resultó muy interesante la parte legal, la última clase. Cuestiones legales de la ONG. [...]. Ahí [en el curso] nos formaron. Porque a veces las cosas están, pero no se sabe. Por ejemplo, el compromiso de voluntariado, la parte legal. Me surgieron un montón de ideas en el curso y después en general vamos viendo y se nos ocurren cosas. Otra clase que me resultó interesante fue la del voluntario, su pertenencia a la institución. Eso lo estamos trabajando y cuesta. Cuando ves esto en el curso es maravilloso porque hay gente que tiene muchas ideas, pero no sabes cómo hacerlo. Bajarlo a la realidad cuesta. (OSC 2) La posibilidad de conocer estrategias para poner en juego buenas ideas.

El programa era muy tentador porque abarcaba los temas donde estábamos flojísimos, y los dividía bien. Nos dio un pantallazo y nos marcó todo lo que teníamos que fortalecer. Todos los puntos donde había que aprender, por ejemplo: captación de fondos, el tema de los padrinos, cómo tener en regla las cuestiones de una ONG, que como te digo, todo esto lo vas haciendo "a los ponchazos" sin tener mucha información, grupos a considerar, etc. Todo nos parecía interesante porque nos servía. Fue superrico el trabajo. (OSC 5) Identificar a partir de un diagnóstico las debilidades organizacionales.

Me di cuenta de eso, de un montón de elementos que faltaban. Eso de tener una visión estratégica, eso para mí fue fundamental. Decir, bueno, cómo puedo implementar un proyecto que contribuya a establecer o a diseñar una estrategia, que finalmente esa estrategia contribuya al logro de la misión de la fundación. (OSC 6) Adquirir una visión estratégica de la organización.

Todos, porque sabíamos que teníamos que organizarnos de una manera mucho más profesional, con una visión mucho más de empresa, de organización. O sea, lo que nosotros tenemos es básicamente un voluntariado muy sólido en cuanto al compromiso con la institución, pero nos faltaba todo lo demás. Y además nos faltaba esto de organizarnos para que no todo el mundo haga todas las cosas. Básicamente era eso. Y además buscar recursos, digamos, al comenzar una organización sin tener la base técnica y las herramientas como para enfrentar el trabajo, solo la voluntad de hacerlo y saber que existe el problema, digamos la voluntad de combatir el problema, el resto de los elementos no los teníamos 
y éramos conscientes de que no los teníamos, entonces, la capacitación era un recurso bueno para nosotros para enfrentar el trabajo de una manera más acertada. (OSC 8) Adquirir conocimientos para profesionalizar el trabajo.

Los nuevos conocimientos que se ponen en juego en este proceso son de tipo "externo" y"formal" (AECA, 2011) para las OSC. Son externos porque el conocimiento es ofrecido por una fuente externa a las organizaciones, $y$ formales porque la innovación es la puesta en marcha de un proceso de capacitación estructurada. Pudo observarse que hubo aprendizaje de contenidos específicos, vinculados con una formación teórico-práctica, es decir, conocimientos que permiten resolver problemas prácticos, estrategias para poner en juego buenas ideas, herramientas específicas con distintos fines (como por ejemplo para implementar proyectos en el territorio), la capacidad de identificar a partir de un diagnóstico las debilidades organizacionales, adquirir una visión estratégica y de liderazgo de la organización. Por otro lado, también se menciona la adquisición de conocimientos de formación general orientados a la profesionalización del trabajo y a mejorar el desempeño en gestión de las organizaciones.

En síntesis, el aprendizaje logrado puede agruparse en conocimientos técnicos específicos, estrategias, nuevos puntos de vista o posicionamientos, y capacidad de diagnosticar las fortalezas y debilidades.

Figura 1. Aprendizajes logrados por las OSC en el marco de la capacitación
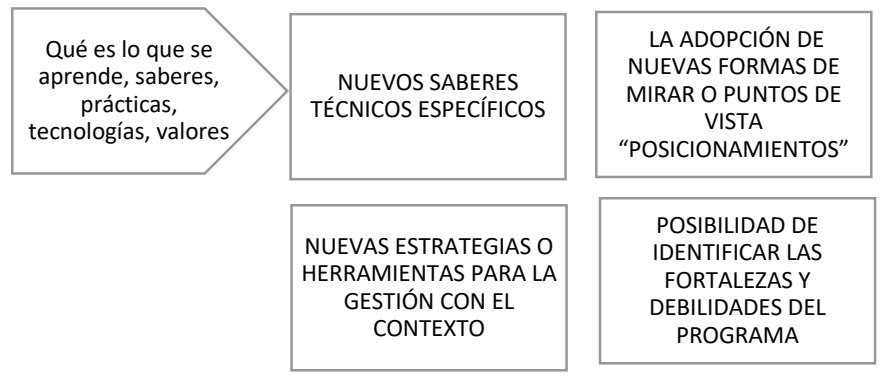

Fuente: reelaborado sobre la base de Fassio y Rutty (2017: 98).

\section{3 ¿Quién aprende? Capacidad de institucionalizar los conocimientos adquiridos}

El aprendizaje organizacional puede analizarse desde la perspectiva de los niveles organizativos implicados, considerando que, según estos, el aprendizaje puede ser prescriptivo o no prescriptivo (AECA, 2011). El aprendizaje prescriptivo se produce cuando solo una parte de la organización aprende; en este caso se dice 
que el proceso de aprendizaje no es completo, ya que solo unos pocos aprenden. Por el contrario, cuando el aprendizaje es no prescriptivo, se produce una integración entre aprendizaje y acción y toda la organización aprende.

Lo que se observó en las conversaciones con los actores consultados es que el ciclo de conocimiento/acción en que se produce el aprendizaje no es completo para toda la organización. La clave a considerar es cómo se disemina el aprendizaje al conjunto de la organización para que este incida en las prácticas habituales (Landaeta, 2003) y el ciclo de aprendizaje organizacional se cierre.

En algunas de las organizaciones estudiadas hubo varios miembros que habían asistido a la capacitación. Sin embargo, en estos casos no todos habían acudido a todos los encuentros. Los conocimientos aprendidos fueron incorporados por las personas y se advirtieron dificultades para su institucionalización, es decir, para que la organización los adoptara e incorporara como parte de su forma de resolver los problemas, a fin de conformar la cultura organizacional.

Yo lo que traía era el material. Yo vengo solamente los lunes, traía el material y lo dejaba acá por si alguno quería leerlo y después preguntarme. (OSC 2) Aprendizaje individual.

En el caso de la universidad, la participación en la puesta en marcha de la innovación generó aprendizajes, aunque no fueron visualizados inmediatamente por los miembros de las cátedras. El aprendizaje puede ser consciente o inconsciente. Es consciente cuando las personas saben que han aprendido, lo que permite el desarrollo de la potencia del aprendizaje; cuando es inconsciente, se estima que su potencial es bajo (AECA, 2011; Gore y Mazzini, 2010). Una situación habitual es que los aprendizajes se incorporan en las organizaciones de manera inconsciente y pasado el tiempo es difícil reconocer su ocurrencia.

Sin embargo, en las entrevistas los docentes manifestaron que entre los aprendizajes realizados observaron la posibilidad de llevar nuevos contenidos al aula vinculados con la práctica de la gestión en organizaciones reales. La experiencia de aprender desde la práctica comporta una nueva forma de enseñar a partir de los casos. La formación de alumnos a través de la práctica se transforma en una nueva actividad pedagógica muy bien valorada. A partir de esta experiencia novedosa, se manifestó también la necesidad de repensar los contenidos del currículum de formación de grado, para incorporar aspectos de la realidad de las organizaciones que no siempre son tenidos en cuenta por la formación.

[... creo que la universidad está un poco distanciada de las OSC [... . Me parece que vamos aprendiendo, vamos mejorando año a año, en mi caso me fortaleció que en mis clases me traigo todos los ejemplos que aprendí en el curso, 
yo se los digo abiertamente; acá aprendo más yo que ustedes porque todos los ejemplos que me comparten o me traen como problemáticas yo después en las clases los doy y los acerco a los chicos a este mundo. Y se vende más el mundo de la empresa, los textos que vemos son de empresas, bueno, hay poco escrito sobre las OSC que usemos en la facultad.

Sí, y esa es una de las cosas que yo llevo a mis clases. Esto de decir que no todo el trabajo es remunerado, y que mucho del trabajo puede ser voluntario, y quizás uno encuentra su realización personal a través del trabajo voluntario. Entonces les acerco ejemplos de organizaciones que por ahí son más cercanas a los chicos, digo, cuando ustedes ven el Banco Alimentario que está al final de la cola del supermercado haciendo una colecta, les acerco ejemplos concretos de organizaciones gestionadas por los jóvenes como Techo, por ejemplo, y cuando puedo también llevo a alguien a las clases a que cuenten de qué se trata la organización. Entonces eso también me transformó a mí en las clases, de flexibilidad a hacer y dar el espacio a la presencia de otro tipo de organizaciones, quizás para difundir esto de que se pueden anotar como voluntarios.

El contacto con estas experiencias genera en los docentes nuevas formas de posicionamiento (Fassio y Rutty, 2017) frente a la tarea, enriqueciendo la práctica y el propio perfil como profesional y como docente.

Eso también me llevó a aprender a que en mi clase también deben tener más presencia otras organizaciones, creo que eso también lo vengo aprendiendo de las OSC. Para darle el lugar que quiero que tengan, al menos en mis clases, ya es algo.

Finalmente, aparece como necesidad el promover la visualización del sector de las OSC como un espacio profesional posible para los egresados de las carreras de la facultad, siendo que, aún hoy, no pareciera constituir un sector reconocido para el futuro de estos profesionales. Por otro lado, los profesores señalan como prioritario que la universidad genere evidencia sobre el fenómeno de las OSC a partir de la investigación con el objeto de iluminar un sector poco estudiado.

\subsection{Los cambios que se hicieron y las estrategias de implementación de lo aprendido en las capacitaciones}

Las organizaciones entrevistadas mencionan cambios relacionados con la planificación de las actividades y de los procesos de sucesión respecto a los siguientes objetivos: transmitir el conocimiento a los miembros que asumirán nuevos roles, el registro de las decisiones y la implementación de bases de datos; elaborar perfiles de voluntarios; materializar iniciativas no concretadas; y poner en mar- 
cha estrategias y sistemas de información para favorecer el trabajo en equipo y estrategias de comunicación sostenidas con sus posibles voluntarios o personas que demandarían sus servicios. En general, estos procesos son vistos como positivos, más allá de las dificultades manifestadas en cuanto a la continuidad de la implementación. Los mayores obstáculos tienen que ver con la falta de recursos económicos, espaciales (algunas no tienen sede propia) y/o de capacidades de sus miembros (para implementar una página web o llevar adelante el registro de la información con un software). La tensión entre hacer como se pueda "con el corazón" y profesionalizar la actividad está presente en los relatos de los entrevistados. La urgencia y la demanda perjudican el sostenimiento de la implementación de las innovaciones, ya que el proceso de transición entre su inclusión como práctica y su institucionalización supone el uso de recursos y tiempo escasos, por lo que, si no se comprende bien la relevancia del cambio, las innovaciones terminan por dejarse de lado y se vuelve "a hacer como siempre se hizo".

Cuando hicimos el curso, nos sirvió también para volcarlo a un papel y decir: "Mensualmente: proyecto - documentación - anotamos esto, esto y esto - contacto, fulanita". Para eso nos sirvió, para armarnos un croquis en papel y después mentalmente para poder todos los meses decir: "Hay que presentar esto". Se internaliza y se trata de que todos los meses se haga eso. (OSC 1) Sistematización de las tareas, con dificultades para sostenerlas.

Después nos dimos cuenta de que no habíamos firmado ningún compromiso de voluntariado. [...] Así que empezamos a implementar este tipo de cosas, y aspectos de sistematización tal vez, de casos, de la interconexión entre psicopedagogas, psicólogas y abogadas. Así que sí, el curso estuvo superbueno. Me resultó muy interesante para ponerlo en práctica... pero me resultó interesante para tener una visión de afuera, ya que esto está hecho a pulmón y remándola, entonces hay cosas que se dificultan. (OSC 2) Formalización del trabajo voluntario / estrategias de articulación entre profesionales voluntarios.

Sí, nuestro sector es de vulnerabilidad social. Desde el punto de vista de discapacidad, hasta que hay personas que no van a poder acceder a nuestros cursos [...]. Hay que ver cómo generar acciones relacionadas con los espacios barriales. No se puede estar ajeno al contexto, pero tampoco podemos abrir un lugar en cada barrio porque no tenemos los recursos. Entonces hay que pensar estrategias, cómo nos articulamos con las instituciones barriales, hacer acciones en los barrios, sin corrernos de los objetivos [...]. En algunas cosas sí. Todo el tiempo estamos renovando capacitaciones, convocatorias, cursos, etc. Así que se hizo todo en función a eso. Lo más importante del curso para mí fue el espacio de intercambio, el contacto con la Facultad. Así se fortalecen instituciones y está 
bueno que te tengan en cuenta. (OSC 3) Articular trabajo en red con otras organizaciones en el territorio.

En lo concreto, armamos una base de datos de todos lo que se ofrecieron como voluntarios en algún momento y esto quedaba en el aire [...]. Otro tema eran los padrinos. Cómo mantener el vínculo sin ser invasivos. Hicimos una gacetilla donde se pasaba el reporte mensual sobre qué había pasado en el mes (con fotos y textos) para que sepan hasta dónde habían llegado sus recursos y de qué manera. (OSC 5) Sistematización de las actividades y difusión de las actividades para sostener los donantes.

Este año lo que hicimos justamente a través de esto, de la proyección y planificaciones, con tiempo [...]. Esto lo hablamos en la comisión y todos dijimos lo mismo, esto fue pactado. Entonces que dijeron: no, pero nosotros no podemos porque no sabemos. (OSC 7) Preparación de la sucesión.

[... ] ahí estamos en eso, que cuando yo empecé averiguar qué es un Community manager, puedes ser alguien joven, que nos maneje la página, y que sea un nexo, y que sea... qué sé yo... Yo no puedo ser, pero tenemos gente, por eso necesitamos gente joven... Y la web ¡va a salir! porque la web... la idea es que, para que nuestro objetivo se cumpla, ser un nexo entre el que necesita un servicio y nosotros que podemos, no ofrecérselos, solamente orientarlos, porque como sabemos quién es un buen cuidador y quién es un atorrante... ya sabemos quiénes, cuáles son los geriátricos clandestinos y cuáles son los geriátricos serios, y qué tiene que reunir un geriátrico y qué no le debe faltar. (OSC 9) Implementando medios de comunicación vía web.

De las cosas más importantes, y que nos dio mucho resultado, fue el tema de poner una persona en Voluntariado que recibiera las ofertas de voluntariado, que hiciera las entrevistas, que se fijara a qué lugar iba cada persona, porque eso nutría a cualquiera de las otras áreas. [... Tenemos un tipo, que es capísimo, que es XXXX, que habla de organización y de las organizaciones de las empresas y nos enseñó a hacer actas de proyecto, que antes: "Qué bueno el acta de proyecto, que lindo", escuchábamos la charla, estaba fantástico, pero después no lo concretábamos. Entonces a partir de eso empezamos a poner más por escrito, hacer más proyectos, a planificar, empezamos a hacer FODA, esos instrumentos que sabíamos que existían, que a lo mejor cada uno en su ámbito profesional los había desarrollado, pero que acá lo hacíamos mucho más a corazón [... ]. Muchas cosas que son organizativas las hicimos a partir de esto. (OSC 9) Materializar: puesta en marcha y operacionalización de iniciativas no concretadas.

Todo esto fue acompañado con el crecimiento de XXX, entonces todos nos dábamos cuenta de que no podíamos estar en todas las funciones, y que era mucho más ordenado que hubiera una persona que se encargara del área de 
voluntariado, que definiera las cosas. [... nos dimos cuenta de que teníamos que dividirnos por áreas como para poder trabajar (OSC 8) Implementación de división de tareas.

Desde la mirada de la universidad, uno de los primeros cambios que supone la innovación es interrumpir la inercia institucional en la que se encontraban las OSC y generar de esta manera un nuevo punto de arranque orientado a identificar formas distintas de resolver problemas. Por otro lado, descubren que existen áreas donde pueden generar mejoras que antes pasaron desapercibidas.

[...] lo primero a lo que ayudó esta formación es a descongelar a las organizaciones, descongelarlas del modo automático en el que estaban [...]. Porque lo he notado en las distintas ediciones en las que participé, cómo impactan los contenidos del curso y actividades que les hacemos hacer en las organizaciones. Porque en general tratamos de que se lleven cosas para hacer o compartir, y ha pasado que gente que no estaba inscripta de la misma organización, en diferentes encuentros viene a participar; porque se enteró por lo que le contó el compañero y como que necesitan hacer masa crítica entre ellos para tratar de generar o plantear esas ideas nuevas. [... ] Este descongelamiento y la adopción de nuevas perspectivas también se vinculan con lo movilizante que resulta acceder a ámbitos universitarios.

Para muchos es shockeante o muy movilizador pisar la universidad, te lo dicen abiertamente: "Nunca creí que en mi vida sin haber terminado el secundario pudiera llegar a la universidad", y lo dicen con un sentido muy especial. Quien ha trabajado con OSC sabe que el "sentir" en esas organizaciones es clave, y cuando ellos sienten eso, sienten que la organización les dio la posibilidad de crecer a ellos personalmente. Me parece que es una transformación y un aprendizaje personal y organizacional; porque ellos después cuando vuelven transmiten que se puede mejorar, sin tener el secundario o sin tener un montón de cosas; entonces eso es transformador, deja su huella; más allá del contenido académico, insisto es importante.

Esta "movilización" se opera en distintos ámbitos de la gestión organizacional y prepara el camino para el logro de sus metas produciendo un mejor desempeño.

\subsection{La conformación de redes organizacionales}

El conocimiento y la interacción con otras organizaciones del territorio se reconocen como un valor relevante y como una innovación en las formas de articulación de la organización. En el discurso de las OSC entrevistadas, se resalta la relación con la Facultad, que acrecienta el capital social de la organización. 
Saber que hay otras instituciones que pasan quizás por lo mismo, en plano básico estoy hablando. Otras instituciones que también tienen problemas financieros, de la luz, del gas, etc. Notar eso, que no somos los únicos. Entonces te sentís "acompañado", porque en realidad después no hay contacto. (OSC 1) Generación de un espacio de contacto que no existía.

$\mathrm{Y}$ el tema de empezar a tirar redes entre diferentes ONG también está bueno. (OSC 2) Generación de un espacio de contacto que no existía.

Bueno, yo trabajo hace muchísimo tiempo en la organización y creo que programas como este son espacios donde uno fortalece un poco su rol y lo que está haciendo. Entonces, estas instancias te corren un poco de lo cotidiano para reflexionar del accionar, por lo que las inquietudes que había tenido estaban relacionadas con esto, con cuestionar varias cosas de la organización. Para mí, algo puntual era el trabajo en red con otras instituciones. Entonces, a veces en nuestros espacios se nos generan muchas relaciones, más allá de que la organización es muy grande y tiene un área de comunicaciones. Uno siempre aprende de la escucha de otros espacios. El intercambio es fundamental. Además, porque nosotros somos una ONG que trabaja en red. Es imposible trabajar sin otros espacios. El conocimiento de ese tipo de instituciones es muy importante. (OSC 3) Integrar una red.

Se cursaba una vez por semana, era superamplio y rico, porque había muchas organizaciones que no conocíamos. Incluso algunas estábamos en el mismo barrio, pero igualmente no nos conocíamos. Tal vez por la falta de recursos para darnos a conocer, lo cual se comparte con las ONG en general: hay mucha voluntad, pero pocos recursos económicos y humanos. (OSC 5) Integrar una red.

Las OSC señalan como estrategia positiva la promoción de dos tipos de relaciones para la mejora de su desempeño: por un lado, la asociación con otras organizaciones de su campo de acción (otras OSC, por ejemplo) trabajo en red, o en alianza y con la propia universidad. Las estrategias de trabajo en red en contextos vulnerables ayudan a las organizaciones a sostener sus iniciativas, a mejorar el desempeño, consolidación y ampliación de sus actividades, y a obtener legitimidad y posibilidad de empoderamiento frente a otros actores sociales (Forni, 2002). Las estrategias de trabajo en alianza pueden servir a varios propósitos, siendo uno de ellos reducir riesgos o asociarse para el crecimiento y la innovación (Scott y Davis, 2015). Asimismo, permiten a las organizaciones con culturas débiles conocer y copiar los procesos de gestión de OSC reconocidas como exitosas en el campo organizacional (Di Maggio y Powell, 1999; Forni y Lucimeire, 2006). 


\section{Conclusiones}

El análisis de la evidencia empírica y la conceptualización a partir de ella dan cuenta del aporte del trabajo con relación a la sistematización del conocimiento sobre el aprendizaje organizacional en el subsector de las OSC en contexto de vulnerabilidad social, así como sobre la sistematización de los aprendizajes de los actores sociales de la universidad pública, campos muy poco estudiados en la Argentina (Schmidt Godoy, De Souza Bido, Bertoia da Silva, Teixeira Maggi da Silva y Reatto, 2015).

La instancia de capacitación teórico-práctica y el contacto con la FCE UNLP, a través de la extensión universitaria, ha supuesto el impulso inicial para realizar un diagnóstico de las debilidades y fortalezas de las OSC en contextos vulnerables (Suárez, 1995), de los procesos de puesta en juego, no sin dificultades, de innovaciones organizacionales y de la aparición de nuevos desafíos a partir de ello, a fin de contribuir al logro de su misión y objetivos. Los aprendizajes organizacionales son relevantes en todas las organizaciones, puesto que se producen a partir de la resolución de los problemas que afrontan y/o a partir de la introducción de innovaciones, pero, en particular en el sector de las OSC que trabajan en contexto y con población vulnerable, son muy importantes, ya que la incorporación de conocimiento y su aplicación supone una mejor utilización de los recursos escasos con los que cuentan (incluyendo las habilidades del voluntariado), el incremento de la calidad y cantidad de su contribución a la resolución de problemas y la mejora de los servicios que brindan a dicha población (Som, Saludin, Shuib, Faisol, Ajis y Nam, 2010).

Las OSC presentan dificultades de gestión en varios campos, en general vinculados con la falta de recursos y una profesionalización incompleta. Muchas de estas razones las acercaron a la universidad en el marco del programa de asistencia técnica desde la extensión. El aprendizaje, a través de una experiencia formal, constituye una forma más de incorporación de conocimiento en las organizaciones. El análisis del recorrido de estos saberes en su vida y los efectos generados constituye un indicador de la ocurrencia de aprendizaje organizacional. Nos preguntamos en qué medida esos aprendizajes transformaron sus prácticas y se incorporaron a la organización como una nueva forma de hacer y resolver problemas. Un primer paso en este camino es la valoración positiva de la experiencia de capacitación por parte de los actores organizacionales, y el reporte de la ocurrencia de algunas transformaciones que son condición necesaria para transformaciones ulteriores, a saber: nuevos saberes técnicos específicos, la adopción de posicionamientos o nuevas formas de mirar, nuevas estrategias para la gestión de los contextos, y el fortalecimiento de las capacidades diagnósticas. 
En algunos casos, estos conocimientos se materializaron en resultados concretos en el campo de la planificación y puesta en marcha de estrategias nuevas de enseñanza. Un ejemplo de ello es la mirada de la universidad, para la cual el proceso de "descongelamiento" y de movilización que genera la experiencia deviene punto de arranque de una forma nueva de hacer en las OSC capacitadas. Ahora bien, la generalización de estos aprendizajes a todos los actores miembros de las OSC será un proceso que requiere mucho tiempo. Sin embargo, ya se observan algunos indicios que muestran el inicio de ese camino.

Por su parte, la universidad también valora la experiencia. El aprendizaje para sí misma se inscribe en el terreno de los nuevos posicionamientos que inspiran nuevas actividades y sujetos de estudio y reflexión de manera permanente en el trabajo con los alumnos, que se sintetiza en la incorporación de nuevos contenidos en el plan de estudios de la licenciatura en Administración.

Dado que la innovación en el marco de actividades en las cuales la universidad traspasa sus muros y contribuye al desarrollo de la sociedad conlleva aprendizaje en múltiples sentidos para todos los actores involucrados (OSC y universidad), resulta de interés documentar estos procesos y multiplicar las prácticas.

\section{Referencias bibliográficas}

Álvarez, M. (2015). "Reflexiones en torno al concepto de innovación y la universidad". En N. F. Lamarra, La innovación en las universidades nacionales. Aspectos endógenos que inciden en su surgimiento y desarrollo (pp. 25 45). Buenos Aires: UNTREF. Recuperado de <https;//www.academia. edu/33472522/La_Innovacion_en_las_Universidad_Nacionales_Aspectos_end\%C3\%B3genos_que_inciden_en_su_surgimiento_y_desarrollo $>$.

Antonacopoulou, E. P. (2006a). "The challenges and Prospects of learningin-practice". Paper presented at the Conference at the University of Warwick, Coventry.

Antonacopoulou, E. P. (2006b). “The relationship between individual and organizational learning. New evidence from managerial learning practices". Management learning, 37 (4), 455-473. doi:10.1177/1350507606070220. Recuperado de <https;//journals. sagepub.com/doi/abs/10.1177/1350507606070220>.

Antonello, S. y Godoy, A. (2010). "A encruzilhada da aprendizagem organizacional: uma visão multiparadigmática". Revista de Administração Contemporânea,14 (2), 310-332.

Argyris, Ch. (1999). Conocimiento para la acción. Una guía para superar los obstáculos del cambio en la organización. Buenos Aires-Barcelona-México: Granica. 
Asociación Española de Contabilidad y Administración de Empresas (AECA) (2011). “Aprendizaje Organizativo”. (21). Recuperado de <httpsः// doi.org/10.1590/S1415-65552010000200008>.

Brandi, U. y Bente, E. (2011). "Organizational learning viewed from a social learning perspective”. En M. Easterby-Smith y M. A. Lyles (eds.) (2011). Handbook of organizational learning and knowledge management. John Wiley $\&$ Sons.

Bustinza, O. F; Molina, L. M. y Arias-Aranda, D. (2010). "Organizational learning and performance: Relationship between the dynamic and the operational capabilities of the firm". African Journal of Business Management, 4 (18), 4067-4078. Recuperado de <https://digibug.ugr. es/bitstream/handle/10481/33179/Bustinza_OrganizationalLearning. pdf? sequence $=1 \&$ is Allowed $=y>$.

Castañeda, Delio Ignacio (2015). "Condiciones para el aprendizaje organizacional". Estudios Gerenciales, 31, n 134 (enero de 2015): 62-67. <https:// doi.org/10.1016/j.estger.2014.09.003. Recuperado de https://www.redalyc. org/pdf/212/21233043007.pdf>.

De Piero, S. (2020)."Organizaciones de la sociedad civil. Tensiones de una agenda en construcción". Recuperado de <https://biblioarchivo.unaj.edu.ar/mostrar/pdf/scvsdf/erwe/e30babda26269cae106109cea7a0d8de0c5141f5 >:

De Souza Minayo, M. C. (2017). "Amostragem e saturaco em pesquisa qualitativa: Consensos e controversias". Revista Pesquisa Qualitativa, 5(7), 1-12.

Di Maggio, Paul J. y Powell, Walter W. (1999). "Retorno a la jaula de hierro: el isomorfismo institucional y la racionalidad colectiva en los campos organizacionales". En El nuevo institucionalismo en el análisis organizacional (pp. 104-195). México: Fondo de Cultura Económica

European Commission (2013). Powering European Public Sector Innovation: Towards A New Architecture. Report of the Expert Group on Public Sector Innovation. Luxemburgo: Publications Office of the European Union. Recuperado de https://ec.europa.eu/futurium/en/content/poweringeuropean-public-sector-innovation-towards-new-architecture-reportexpert-group

FAssio, A. (2007). Organizaciones voluntarias de personas mayores: entre la exclusión y la inclusión social. Tesis de doctorado, Facultad de Filosofia y Letras, Universidad de Buenos Aires. Recuperado de <http://repositorio.filo.uba.ar/handle/ filodigital/1279>.

Fassio, A. y Rutty, M. G. (2017). “Gestionando el aprendizaje en organizaciones que educan en el marco de una innovación social en el campo de atención 
sociosanitaria”. RELAPAE (7), pp 81-95. ISSN 2408-4573 Recuperado de <http://revistasuntref.com.ar/index.php/relapae/article/view/26/66>:

Fassio, A. y Rutty, M. G. (2020). "El aprendizaje organizacional ¿una moda?, ¿una nueva forma de llamar un viejo fenómeno?, ¿ ¿ un elemento permanente de la vida de las organizaciones? Avances en el campo de estudio". En M. L. Pagani y M. Manuele (coordinadores). La mirada organizacional. Enfoques y metodología para el análisis (pp. 159-185). Editorial de la UNLP. ISBN 978987-8348-49-0. <http://sedici.unlp.edu.ar/handle/10915/102504>.

Forni, P. (2002). "Las Redes Inter-Organizacionales y sus implicancias en el desarrollo de las Organizaciones Comunitarias de los Pobres y Excluidos". Primer Congreso Nacional de Políticas Sociales, Universidad Nacional de Quilmes - Asociación Argentina de Políticas Sociales. Bernal, Quilmes, Argentina, 30-1/mayo 2002. Recuperado de <https://racimo.usal.edu. ar/95/1/Forni4.pdf $>$ :

Forni, P. (2010). Los estudios de caso: Orígenes, cuestiones de diseño y sus aportes a la teoría social, 5, 1-26.

Forni, P. (2012). "De Bacon y Mill a la lógica difusa. La investigación comparativa en Ciencias Sociales". Prisma social. Revista de ciencias sociales, 29-56.

Forni, P.; Castronuovo, L. y Nardone, M. (2012). "Las organizaciones en red y la generación de capital social. Implicancias para el desarrollo comunitario". Miríada. Investigación en Ciencias Sociales, (8), 79-106.

Forni, P. y Lucimeire, V. L. (2006). "El desarrollo y legitimación de las organizaciones del tercer sector en la Argentina. Hacia la definición de un isomorfismo periférico". Sociologías, 16, 216-249. Recuperado de <httpsः//www.scielo. br/scielo.php?pid=S1517-45222006000200009\&script $=$ sci_arttext $>$.

Gerometta, J; Haussermann, H. y Longo, G. (2005). "Social innovation and civil society in urban governance: strategies for an inclusive city". Urban Studies, 42 (11), 2007-2021. Recuperado de <https://journals.sagepub.com/ doi/abs/10.1080/00420980500279851>.

Gore, E. (2003). Conocimiento colectivo. Buenos Aires: Ediciones Granica.

Gore, E+y Mazzini, M. (2010). Hacer visible lo invisible. Buenos Aires: Granica. Grunwald, A. (2011). "Responsible innovation: bringing together technology assessment, applied ethics, and STS research". Enterprise and Work Innovation Studies, 7, 9-31. Recuperado de <https://run.unl.pt/handle/10362/7944>.

LAndaeta, R. P. (2003). "Gestión del conocimiento: una visión integradora del aprendizaje organizacional". Revista de investigación en Gestión de la Innovación y Tecnología. Conocimiento e Innovación (18) 52-58. Recuperado de <https:// www.researchgate.net/profile/Jesus_Tejedor/publication/28063582_Com- 
paracion_de_los_sistemas_de_ciencia_y_tecnologia_existentes_en_Espana/links/552690e10cf2628d5afedabb/Comparacion-de-los-sistemas-deciencia-y-tecnologia-existentes-en-Espana.pdf\#page $=52>$.

Liebler, C.y Ferri, M. (2004). NGO networks; building capacity in a changing world. Report from a study supported by the USAID Bureau for Democracy, Conflict and Humanitarian Assistance. Washington, DC: USAID, Office of Private and Voluntary Cooperation. Recuperado de <http://wikiciv.org.rs/ images/9/9a/NGO_Networks.pdf>.

Longo, M. E. y Forni, P. (2004)."Las respuestas de los pobres a la crisis. Redes de Organizaciones comunitarias y la búsqueda de soluciones a los problemas de las áreas periféricas de Buenos Aires". En Argentina ¿Qué perspectivas económicas, politicas y sociales para la democracia después de diciembre de 2001? (pp. 70-97). UNESCO. <https://www.aacademica.org/pforni/13>:

Moulaert, F* y Sekia, F* (2003). "Territorial innovation models: A critical survey”. Regional Studies, 37 (pp. 289-302). Recuperado de <https://www.researchgate. net/profile/Frank_Moulaert/publication/24087717_Territorial_Innovation_ Models_A_Critical_Survey/links/0912f50c5196d963e2000000.pdf >.

Neiman, G. y Quaranta, G. (2007). "Los estudios de caso en la investigación sociológica”. En I. Vasilachis de Gialdino (coord.). Estrategias de Investigación Cualitativa (pp. 213-234). Buenos Aires: Gedisa.

Perlo, C. (2011). "Procesos de cambio colectivo, nuevos marcos de comprensión para aprender el holomovimiento". Enl@ce: Revista venezolana de Información, Tecnología y Conocimiento, 81-96. Recuperado de <https://www.redalyc.org/ pdf/823/82317684006.pdf $>$.

Rodríguez, A. M. E. y Cedeño, B. E. (2012). "La innovación entre dos manos: la invisible del mercado y la visible del Estado”. TRILOGÍA. Ciencia, Tecnología y Sociedad, 6, 13-30. <https;//revistas.itm.edu.co/index.php/trilogia/ article/view/33>:

San Martín Cantero, D. (2014). "Teoría fundamentada y Atlas. ti: recursos metodológicos para la investigación educativa". Revista electrónica de investigación educativa, 16 (1), 104-122. Recuperado de <http‡//www.scielo.org.mx/ scielo.php?script $=$ sci_arttext $\&$ pid $=$ S1607-40412014000100008>.

Schmidpeter, R. (2013). "Social Innovation: A New Concept for a Sustainable Future?". En T. Osburg y R. Schmidpeter (eds.). Social Innovation. Solutions for a Sustainable Future (pp. 1-12). Berlín: Springer. Recuperado de <https://isfcolombia.uniandes.edu.co/images/2020-20/Semana_10/ Social_Innovation_Solutions.pdf $>$ : 
Schmidt Godoy, A.; De Souza Bido, D.; Bertoia da Silva, N.; Teixeira Maggi da Silva, P. y Reatto, D. (2015). "O campo da aprendizagem organizacional no Brasilः uma revisão multiparadigmática da produção de 2006 a 2012”. XVIII SemeAd, Seminários de Administração, noviembre. Recuperado de: <https://www.researchgate.net/publication/309158944>.

Sсотт, W. R. y Davis, G. F. (2015). Organizations and organizing: Rational, natural and open systems perspectives. Nueva York: Routledge.

Senge, P. (2005). La Quinta Disciplina. Cómo impulsar el aprendizaje en la organización inteligente. Barcelona: Granica.

Som, H.; Saludin, M. N.; Shuib, S.; Faisol, M.; Ajis, M. N. y Nam, R. Y. T. (2010). "Learning organization elements as determinants of organizational performance of non-profit organizations (NPOs) in Singapore". International NGO Journal, vol. 5(5) (pp. 117-128). Recuperado de: <http // www.academicjournals,org/INGOJ>.

Stake, R. (2013). "Estudios de caso cualitativos”. En N. Denzin y Y. Lincoln (eds.). Manual de investigación cualitativa. Estrategias de investigación cualitativa, vol. III (pp. 155-197). Buenos Aires: Gedisa

SuÁrez, F. (1995). "Debilidades de las ONG”. Revista Enoikos, (3) 9, 45-53.

Sulbrandt, J.; Navarrete, N. y Piergentilli, N. (2007). "Formas sociales que facilitan la entrega de servicios sociales". En J. C. Cortázar Velarde (ed.). Entre el diseño y la evaluación: el papel crucial de la implementación de los programas sociales (pp. 119-165). Washington: IADB. Recuperado de: $<$ https://publications.iadb.org/es/publicacion/14195/entre-el-diseno-y-laevaluacion-el-papel-crucial-de-la-implementacion-de-los $>$.

Vera, D.; Crossan, M. y Apaydin, M. (2011). "A framework for integrating organizational learning, knowledge, capabilities, and absorptive capacity". En Handbook of organizational learning and knowledge management (pp. 153-180).

Wenger, E. (2000). "Communities of Practice and Social Learning Systems". Organization 7(2), 225-246. Recuperado de: <https://www.psycholosphere.com/Communities\%20of\%20Practice\%20\&\%20Social\%20Learning\%20 Systems\%20by\%20Wenger\%202000.pdf>. 